\title{
Clinical Study Skin Resistivity Value of Upper Trapezius Latent Trigger Points
}

\author{
Elżbieta Skorupska, ${ }^{1}$ Jarosław Zawadziński, ${ }^{2}$ \\ Agata Bednarek, ${ }^{1}$ and Włodzimierz Samborski ${ }^{1}$ \\ ${ }^{1}$ Department of Rheumatology and Rehabilitation, Poznan University of Medical Sciences, 28 Czerwca 1958r., 60-545 Poznań, Poland \\ ${ }^{2}$ Department of Bionics and Bioimpedance, Poznan University of Medical Sciences, Parkowa 2, 60-775 Poznań, Poland
}

Correspondence should be addressed to Elżbieta Skorupska; skorupska@ump.edu.pl

Received 19 December 2014; Revised 3 June 2015; Accepted 8 June 2015

Academic Editor: Matteo Paci

Copyright (C) 2015 Elżbieta Skorupska et al. This is an open access article distributed under the Creative Commons Attribution License, which permits unrestricted use, distribution, and reproduction in any medium, provided the original work is properly cited.

Introduction. The skin resistivity (SkR) measurement is commonly recommended for acupoints measurement, but for trigger points (TrPs) only one study is available. The purpose of the study was to evaluate SkR for latent TrPs compared to non-TrPs and the surrounding tissue. Material and Methods. Forty-two healthy volunteers with unilateral latent upper trapezius TrPs (12 men, 30 women) aged 21-23 (mean age: $22.1 \pm 0.6 \mathrm{y}$ ) participated in the study. Keithley electrometer 610B was used for measuring SkR (Ag/AgCl self-adhesive, disposable ground electrode: $30 \mathrm{~mm}$ diameter). SkR was measured for latent TrPs and compared to opposite non-TrPs sites and the surrounding tissue. Results. The SkR decrease of TrPs-positive sites as compared to TrPs-negative sites and the surrounding tissue was confirmed. However, no statistically significant difference in the SkR value occurred when all data were analyzed. The same was confirmed after gender division and for TrPs-positive subjects examined for referred pain and twitch response presence. Conclusion. SkR reactive changes at latent TrPs are possible but the results were not consistent with the previous study. Thus, caution in applying SkR to latent TrPs isolation is recommended and its clinical use should not be encouraged yet. Further studies, especially on active TrPs, are yet required.

\section{Introduction}

Myofascial pain syndrome (MPS) is defined as a local pain syndrome characterized by (i) trigger points (TrPs), that is, limited sites of severe muscle tenderness or hypersensitivity, and (ii) a determined area of referred pain [1-3]. Trigger points are divided into latent and active, and the only differences between them are spontaneous pain characteristic of active points and the level of biochemicals. The incidence of $\mathrm{TrPs}$ is very common in general population, and the prevalence in around $30 \%$ of pain patients consulting in primary care has been proved [4].

The diagnosis of TrPs is based on essential clinical criteria (taut band, tender knot, pain recognition, and restricted range of motion) defined by Travell and Simons. Additionally, when one or more confirmatory signs such as referred pain, twitch response, or spontaneous electrical activity are confirmed, the diagnosis is more valid [3]. For research purposes, the most commonly tested muscle is the upper trapezius because of the high prevalence of TrPs in that muscle and easy access to the taut band $[5,6]$. Moreover, Barbero et al. claimed that an experienced physiotherapist can reliably identify $\operatorname{TrP}$ locations in the upper trapezius muscle using a palpation protocol [7].

Over the last past years, a significant growth of interest in trigger points has been observed among researchers due to the objective confirmation of TrPs presence. The biopsy of the area defined as a trigger point has shown partial shortening and contraction of sarcomeres in particular muscle fibers, described as a "large, round, and dark muscle fiber." This causes a statistically significant increase in the average myocyte diameter [8], confirmed recently by magnetic resonance elastography and ultrasonography [912]. Unfortunately, these techniques are not easily applicable to clinical practice at this time. That is why the diagnosis of TrPs is still based on palpatory diagnostic criteria and an easy and cheap method for TrPs confirmation is still required [3].

In the vicinity of TrPs, a deregulated motor end plate sustained by a neural loop of sensory and autonomic afferents in the central part of a TrP was confirmed. However, there 
is no overlap between TrPs and the innervation zone [13]. Additionally, Shah et al. [14] proved the presence of $\mathrm{H}+$, BK, CGRP, SP, TNF-, IL-1, serotonin, and norepinephrine in active TrPs only. Electromyography has shown a spontaneous electrical activity (SEA) at TrPs during electrical silence of adjacent muscle fibers. SEA is defined as minute loci within TrPs that produce characteristic low-amplitude electrical activity $[15,16]$. This indicates a direct relationship with bioelectric measurement and the possibility of using skin resistance as a noninvasive and easy TrPs measurement.

There is only one study that postulates the meaning of skin resistance used as a measurement tool for TrPs isolation from the surrounding tissue [17]. Moreover, based on that study, it seems useless to compare the value of TrPs skin resistivity to control group (healthy subjects). The average value of human resistance is $1500 \mathrm{ohms}$, but it varies greatly for different people and the results may be different even for one person due to many intrinsic and extrinsic factors. Because of this, the lateral presence of TrPs should be an inclusion criterion, with the opposite site corresponding to the common localization of TrPs becoming the control. Moreover, because it was proven that depending on gender skin resistance values for healthy subjects differ, skin resistivity should be investigated regarding gender [18].

The purpose of the study was to determine the SkR value of TrPs within the upper trapezius muscle compared to non-TrPs (control) and the surrounding tissue (norm). Additionally, the influence of gender differences and TrPs confirmatory signs on skin resistivity measurement was examined.

\section{Material and Methods}

2.1. Ethics Statement. The study protocol was approved by the Institutional Review Board of the Poznan University of Medical Sciences (number 790/12). The trial was registered in the Australian New Zealand Clinical Trials Registry: ACTRN12614001169639. Before their participation, all subjects were thoroughly informed of the methods and procedures used and gave their written consent to participate in this institutionally approved study carried out in accordance with the Declaration of Helsinki.

2.2. Patients. Forty-two healthy volunteers (12 men, 30 women) at the age of 21-23 (mean age: $22.1 \pm 0.6 \mathrm{y}$ ) participated in the study. All volunteers participating in the trial filled in the clinical health questionnaire. The inclusion criteria were unilateral latent trigger points within the upper trapezius muscle. The exclusion criteria were (i) rheumatologic or neurological diseases and other serious medical conditions and real pain problems of the shoulder girdle and neck (because of the measurement methods of electrodermal skin resistance); (ii) surgery or/and posttraumatic incidence of the upper extremity and neck; (iii) diabetic problems; (iv) current use of some pain killers and other pharmacotherapies; (v) dermal problems of the upper back skin.

2.3. Trigger Points Confirmation. Before the main experiment, the participants were reexamined with respect to the

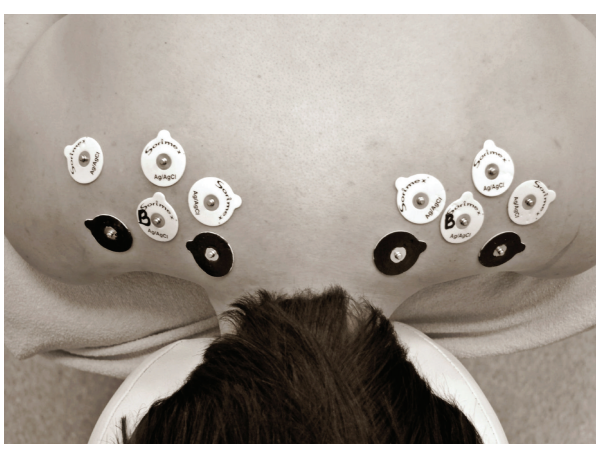

Figure 1: Example of TrPs, control (TrPs-negative), and norm electrodes localization during SkR measurement. The black electrodes show TrPs localization ( $\mathrm{x} 1$ and $\mathrm{x} 2$ ) and, on the opposite site, next two black electrodes TrPs negative $\mathrm{x} 1$ and $\mathrm{x} 2$ localization. The electrodes with the letter " $B$ " show "reference points," and the three white electrodes on each side refer to the norm (not tender points of the surrounding tissue).

presence of latent trigger points within the upper trapezius muscle (both left and right) defined by Travell and Simons as $\mathrm{x} 1$ and $\mathrm{x} 2$. The diagnosis was made by a clinician experienced in myofascial pain diagnosis according to Travell and Simons' criteria [3]. The confirmatory signs, namely, referred pain and twitch response, were examined. For the purposes of skin resistance measurement, each of the latent $\mathrm{TrPs}$ was marked and defined as TrPs-positive. The same was done for the area corresponding to the region common for TrPs with negative results named as non-TrPs (control). Then the examiner looked for four pain-free points on each side (not tender, without any features of TrPs) in the closest area to the previously marked crosses. When the four points were found, they were marked and named norm.

2.4. Skin Resistance Measurement. Experiments were performed with the subjects placed prone in a quiet room with the ambient temperature set at $23 \pm 1^{\circ} \mathrm{C}$. They rested for $15 \mathrm{~min}$. before each trial.

The participants were evaluated towards skin resistance in the marked area by an expert without any knowledge about TrPs examination results. During tests, a Keithley electrometer $610 \mathrm{~B}$ was used for measuring skin resistance (Ag/AgCl self-adhesive, disposable ground electrode, $30 \mathrm{~mm}$ diameter). The electrodes were placed on the specific points of the skin. Two electrodes were put on the points defined as $\mathrm{x} 1$ and $\mathrm{x} 2 \mathrm{TrPs}$ within the upper trapezius (TrPs-positive and control) and four on other points showing no evidence of pathology (norm) (Figure 1). One of the norm points (positioned centrally in relation to the other) functioned as a reference point. Skin resistance (in ohms, $[\Omega]$ ) was measured once in every marked point. The electrometer was rezeroed before every trail. Input parameters were turned off and the electrometer was corrected for internal noise to recalibrate as the participants changed. All tests were completed in a climate controlled room.

2.5. Data Analysis. Resistance values previously obtained from each of the marked points (TrPs-positive, control, 
and norm) were used to calculate the resistivity using the following formula:

$$
\rho=\frac{R S}{x},
$$

where $\rho$ is resistivity $[\Omega \mathrm{m}] ; R$ is resistance $[\Omega] ; S$ is electrode surface $\left[\mathrm{m}^{2}\right] ; x$ is distance between electrodes (reference and others) $[\mathrm{m}]$.

The mean value obtained from the calculations made for three norm points was determined in order to calculate the so-called personal norm for the pain-free area (without any $\operatorname{TrPs}$ features). Additionally, $\operatorname{TrPs}$ skin resistivity values were analyzed with regard to the gender and the presence of confirmatory signs (referred pain and twitch response).

2.6. Statistical Analysis. The two-tailed $t$-tests were performed in order to ensure that data are representative of the full population of possible data values. In order to compare the differences in skin resistivity values of TrPs-positive and TrPs-negative sites as compared to their control points, the two-tailed $t$-tests were applied. All of the above dependences with regard to gender division, referred pain, and twitch response presence were tested by the two-tailed $t$-test. Values in the text, figures, and tables are expressed as \pm standard error of the mean (SEs). All levels of probability were set at a significant level of 0.05 . The statistical analysis was performed using IBM SPSS Statistics, version 20.

\section{Results}

The skin resistivity decrease of TrPs-positive sites compared to other measured sites, that is, TrPs-negative (control) and norm, was confirmed. However, the two-tailed $t$-tests showed no statistical significance for skin resistivity between TrPspositive to norm $(p=0.59)$, TrPs-positive to TrPs-negative (control) $(p=0.19)$, and TrPs-negative (control) to norm ( $p=0.12$ ) when all data were analyzed. Figure 2 presents the mean value of skin resistivity for TrPs-positive and TrPsnegative compared to their norm. After rejecting markedly different values, the same tendency was confirmed.

After sex division, a contrary tendency of skin resistivity value was observed: among women, SkR decrease of TrPs sites compared to the surrounding tissue (norm), and for men, SkR increase for TrPs compared to norm (Figure 3). For both subgroups, the two-tailed $t$-tests confirmed no significant changes in skin resistivity between TrPs-positive to the surrounding tissue (norm) (women $p=0.07$; men $p=0.56$ ) and TrPs-negative (control) (women $p=0.23$; men $p=0.34$ ) after sex division. After rejecting markedly different values, the same tendency was confirmed.

3.1. Skin Resistivity of TrPs Depending on Presence of Confirmatory Signs. For TrPs-positive subjects, skin resistivity values were analyzed with regard to referred pain and twitch response occurrence. There was no statistical difference between TrPs-positive sites with referred pain as compared to their norm $(p=0.17)$ and with regard to the presence of twitch response compared to their norm $(p=0.11)$

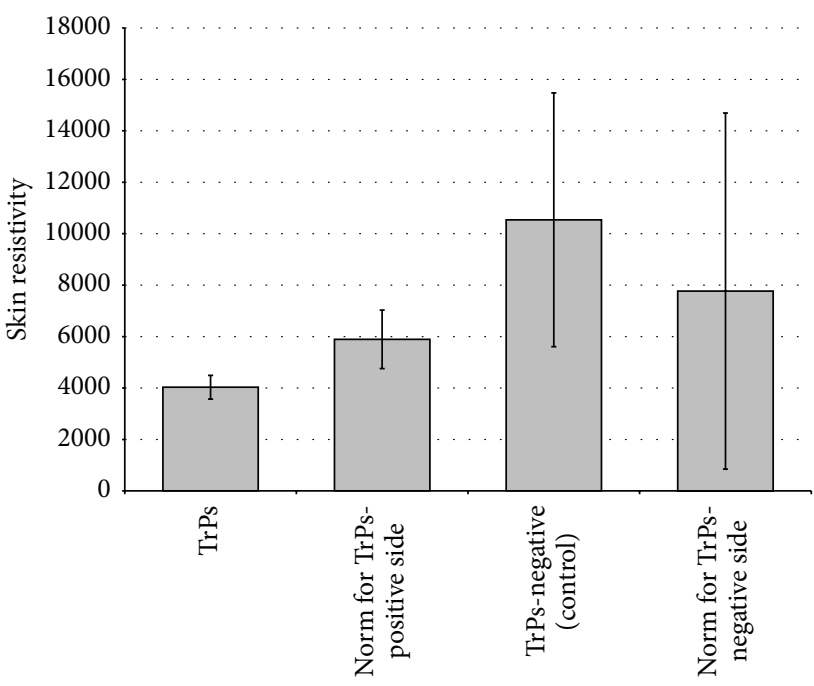

FIgURe 2: Mean value of skin resistivity for TrPs-positive and TrPsnegative compared to their norm.

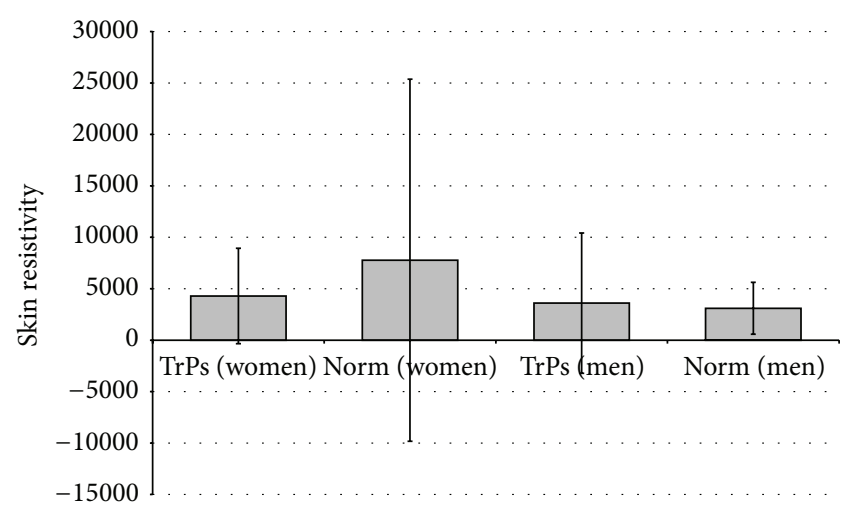

FIgURE 3: Mean value of skin resistivity for TrPs-positive compared to the surrounding tissue (norm) after sex division.

(Figure 4). The same was confirmed after sex division for referred pain: men: $3917.9 \pm 7367.0$ versus $2915.7 \pm 1953.9 \Omega *$ $\mathrm{m}(p=0.31)$; women: $4404.4 \pm 4800.1$ versus $8091.7 \pm$ $18413.2 \Omega * \mathrm{~m}(p=0.08)$. When twitch response presence was analyzed, for men it is $3335.2 \pm 7729.7$ versus $3483.8 \pm$ $2861.7 \Omega * \mathrm{~m}(p=0.91)$; women $4613.1 \pm 5684.6$ versus $10067.7 \pm 22783.5 \Omega * \mathrm{~m}(p=0.10)$. Confirmatory signs, namely, twitch response and referred pain, do not significantly differentiate skin resistivity of TrPs-positive with/without confirmatory signs.

\section{Discussion}

The main result of the present study is that skin resistivity (SkR) measurement does not significantly differentiate latent trigger point localization from the surrounding tissue and asymptomatic region corresponding to the common localization of TrPs (control) (Figure 2). This is contrary to the study by Shultz et al. [17] who recommended skin resistance measurement for trigger points (TrPs) confirmation. However, 


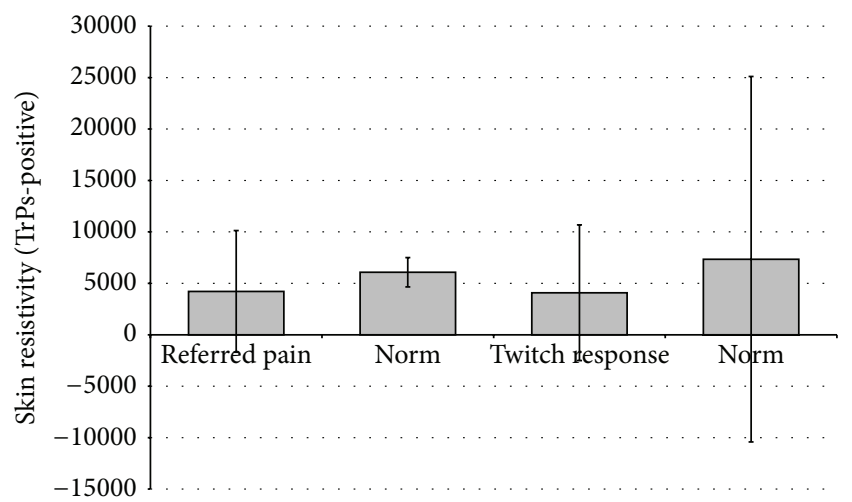

FIgURE 4: Mean value of TrPs skin resistivity dependent on the confirmatory sign presence.

a similar tendency of the SkR value at TrPs site, namely, SkR decrease, compared to the surrounding tissue, was confirmed in both studies. That tendency seems valid for future studies because skin resistance decrease in conditions related to the sympathetic nervous system (SNS) activation was suggested $[19,20]$. Interestingly, the latest data indicate the meaning of SNS in TrPs pain propagation [21-25]. However, it is not known whether SNS activity influences all TrPs or only severe active TrPs subjects. If we assume that only severe state of active TrPs is SNS-related, then only such cases can be SkR sensitive. However, no objective method of active TrPs division exists and, thus, it remains just an assumption.

Additionally, the distribution of standard deviations in the present study indicates that it is impossible to establish TrPs resistance norm range which could be later used in practical assessment of patients. There may be several reasons for this situation. Skin resistance value depends on many factors such as blood flow, thickness, and sweat glades activity. These factors can provoke constant changes during the day, which may consequently lead to skin resistance changes [26]. Thus, it is difficult to establish resistance norm for skin in vivo. Similar observations were proven for acupuncture studies where due to high variability the results were assessed as relative rather than absolute numbers [27].

Furthermore, the difference between the present and Shultz et al.s [17] studies can be explained by a certain methodological issue. Some information within the discussion by Shultz et al. indicated diminished credibility. The authors stated that false-negative TrPs examination would make the difference between skin resistance values of nonTrPs and experimental group negligible [17]. However, trigger points diagnosis within the upper trapezius muscle is one of the easiest and, since reliable identification using a palpation protocol was proven, there should be no doubts regarding TrPs confirmation [7].

Additionally, the differences in results of undertaken studies may be explained through different methodologies that were applied to experiments. First of all, in the present study, AG/Cl electrodes were used in every measured point. Shultz et al. [17] used ultrasound gel and put a metal electrode to skin using the gel. The only ground electrode was $\mathrm{Ag} / \mathrm{Cl}$ electrode. The great disadvantages of this method are differences in contact area (in the case of gel and electrode), as well as differences in electrode application pressure [28]. Secondly, we located a ground electrode in the center of the measurement area and tried to remain constant distance between measured points and the ground electrode. It is known that the location has significant influence on measurements because resistance depends on distance between electrodes [29]. Shultz et al. did not define the location of the ground electrode [17]. However, after the data presented is analyzed, it can be assumed that the ground electrode was located a few centimeters to the left from the measurement area. The methodological differences, including technical standards which influenced the study results, were indicated by others and are observed in the TrPs resistance study discussed above [30].

Another interesting aspect of the present study is data analysis after gender division. This was recommended in many studies because of the differences in skin thickness (greater in man) and skin blood flow (much greater in man), which influence skin as an electric conductor $[18,31,32]$.

In the light of the present study that recommendation revealed interesting observation, data analysis after gender division in the present study showed a completely opposite reaction of SkR in the TrPs region, namely, a decrease or an increase depending on the sex (Figure 3). This situation may be explained by differences in sweat glands localization and mode of action; while the density of sweat glands in men is lower, in women sweating reaction is delayed and is less copious, which may influence electrodermal activity results [33].

Shultz et al. [17] explained their results and recommended the usefulness of TrPs skin resistance measurement basing on Shah's findings [14], which confirmed the existence of a localized hypoxic region in $\operatorname{TrPs}$ and a local increase in sensitizing substances in that area possibly inducing locally greater blood flow and secretion from sweat glands via stimulation of the autonomic nervous system. Shultz et al. [17] speculated that twitch response could increase the autonomic response which would cause changes to vasodilation and sweat secretion to the localized area. However, Shultz et al. did not analyze the meaning of twitch response presence, which was a differentiating factor in Shah's study [14]. The data in the present study confirmed that for TrPs localization there is no SkR difference dependent on the presence of twitch response or referred pain. However, maybe the SkR measurement just after needle-evoked twitch response could reveal some differences. Also perhaps it is not the twitch response presence but the severity of TrPs (active or latent form) that is important for the SkR outcome. Moreover, there are only two studies concerning SkR measurement, both based on the same muscle. Thus, other muscles with TrPs should be evaluated towards the SkR value compared to the surrounding tissue. Further studies concerning this idea are recommended in the future.

\section{Conclusion}

SkR reactive changes at latent $\mathrm{TrPs}$ are possible but the results were not consistent with the previous study. Thus, caution in 
applying SkR to latent TrPs isolation is recommended and its clinical use should not be encouraged yet. Further studies, especially on active TrPs, are yet required.

\section{Conflict of Interests}

The authors declare no conflict of interests.

\section{Authors' Contribution}

Elżbieta Skorupska contributed to the conception and design of the work, acquisition of data, interpretation of data and drafting the paper; Jarosław Zawadziński helped with acquisition of data, analysis of data, and drafting the paper; Agata Bednarek contributed to interpretation of data; Włodzimierz Samborski helped with final approval of the version to be published. All the authors listed have contributed substantially to the paper.

\section{Acknowledgments}

The authors thank Malgorzata Mankowska and Richard Ashcroft who provided proofreading services.

\section{References}

[1] J. Travell and S. H. Rinzler, "The myofascial genesis of pain," Postgraduate Medicine, vol. 11, no. 5, pp. 425-434, 1952.

[2] J. R. Fricton, "Myofascial pain syndrome: characteristics and epidemiology," in Advances in Pain Research and Therapy: Myofascial Pain and Fibromyalgia Syndrome, J. R. Fricton and E. Awad, Eds., vol. 17, pp. 107-127, Raven Press, New York, NY, USA, 1990.

[3] J. G. Travell and D. G. Simons, Eds., Myofascial Pain and Dysfunction: The Trigger Point Manual, Williams \& Wilkins, Baltimore, Md, USA, 1983.

[4] S. A. Skootsky, B. Jaeger, and R. K. Oye, "Prevalence of myofascial pain in general internal medicine practice," Western Journal of Medicine, vol. 151, no. 2, pp. 157-160, 1989.

[5] M. Ziaeifar, A. M. Arab, N. Karimi, and M. R. Nourbakhsh, "The effect of dry needling on pain, pressure pain threshold and disability in patients with a myofascial trigger point in the upper trapezius muscle," Journal of Bodywork and Movement Therapies, vol. 18, no. 2, pp. 298-305, 2014.

[6] C. Fernández-de-las-Peñas, H. Y. Ge, L. Arendt-Nielsen, M. L. Cuadrado, and J. A. Pareja, "Referred pain from trapezius muscle trigger points shares similar characteristics with chronic tension type headache," European Journal of Pain, vol. 11, no. 4, pp. 475-482, 2007.

[7] M. Barbero, P. Bertoli, C. Cescon, F. Macmillan, F. Coutts, and R. Gatti, "Intra-rater reliability of an experienced physiotherapist in locating myofascial trigger points in upper trapezius muscle," Journal of Manual and Manipulative Therapy, vol. 20, no. 4, pp. 171-177, 2012.

[8] S. Mense and D. G. Simons, Muscle Pain: Understanding Its Nature, Diagnosis, and Treatment, Lippincott Williams \& Wilkins, Philadelphia, Pa, USA, 2001.

[9] Q. Chen, J. Basford, and K. N. An, "Ability of magnetic resonance elastography to assess taut bands," Clinical Biomechanics, vol. 23, pp. 623-629, 2008.
[10] Q. Chen, S. Bensamoun, J. R. Basford, J. M. Thompson, and K.-N. An, "Identification and quantification of myofascial taut bands with magnetic resonance elastography," Archives of Physical Medicine and Rehabilitation, vol. 88, no. 12, pp. 16581661, 2007.

[11] S. Sikdar, R. Ortiz, T. Gebreab, L. H. Gerber, and J. P. Shah, "Understanding the vascular environment of myofascial trigger points using ultrasonic imaging and computational modeling," in Proceedings of the 32nd Annual International Conference of the IEEE Engineering in Medicine and Biology Society (EMBC '10), vol. 1, pp. 5302-5305, September 2010.

[12] S. Sikdar, J. P. Shah, T. Gebreab et al., "Novel applications of ultrasound technology to visualize and characterize myofascial trigger points and surrounding soft tissue," Archives of Physical Medicine and Rehabilitation, vol. 90, no. 11, pp. 1829-1838, 2009.

[13] M. Barbero, C. Cescon, A. Tettamanti et al., "Myofascial trigger points and innervation zone locations in upper trapezius muscles," BMC Musculoskeletal Disorders, vol. 14, article 179, 2013.

[14] J. P. Shah, J. V. Danoff, M. J. Desai et al., "Biochemicals associated with pain and inflammation are elevated in sites near to and remote from active myofascial trigger points," Archives of Physical Medicine and Rehabilitation, vol. 89, no. 1, pp. 16-23, 2008.

[15] D. R. Hubbard and G. M. Berkoff, "Myofascial trigger points show spontaneous needle EMG activity," Spine, vol. 18, no. 13, pp. 1803-1807, 1993.

[16] D. G. Simons, C.-Z. Hong, and L. S. Simons, "Endplate potentials are common to midfiber myofacial trigger points," The American Journal of Physical Medicine and Rehabilitation, vol. 81, no. 3, pp. 212-222, 2002.

[17] S. P. Shultz, J. B. Driban, and Ch. B. Swanik, "The evaluation of electrodermal properties in the identification of myofascial trigger points," Archives of Physical Medicine and Rehabilitation, vol. 88, no. 6, pp. 780-784, 2007.

[18] E. Tur, "Physiology of the skin-differences between women and men," Clinics in Dermatology, vol. 15, no. 1, pp. 5-16, 1997.

[19] C. P. Richter and D. T. Katz, "Peripheral nerve injuries determined by the electrical skin resistance method," The Journal of the American Medical Association, vol. 122, no. 10, pp. 648-651, 1943.

[20] L. H. Riley Jr. and C. P. Richter, "Use of electrical skin resistance method in the study of patients with neck and upper extremity pain," Johns Hopkins Medical Journal, vol. 137, no. 2, pp. 69-74, 1975.

[21] H.-Y. Ge, C. Fernández-de-las-Peñas, and L. Arendt-Nielsen, "Sympathetic facilitation of hyperalgesia evoked from myofascial tender and trigger points in patients with unilateral shoulder pain," Clinical Neurophysiology, vol. 117, no. 7, pp. 1545-1550, 2006.

[22] Y. Kimura, H.-Y. Ge, Y. Zhang, M. Kimura, H. Sumikura, and L. Arendt-Nielsen, "Evaluation of sympathetic vasoconstrictor response following nociceptive stimulation of latent myofascial trigger points in humans," Acta Physiologica, vol. 196, no. 4, pp. 411-417, 2009.

[23] Y. Zhang, H.-Y. Ge, S.-W. Yue, Y. Kimura, and L. ArendtNielsen, "Attenuated skin blood flow response to nociceptive stimulation of latent myofascial trigger point," Archives of Physical Medicine and Rehabilitation, vol. 90, no. 2, pp. 325-332, 2009.

[24] E. Skorupska, M. Rychlik, W. Pawelec, A. Bednarek, and W. Samborski, "Trigger point-related sympathetic nerve activity in 
chronic sciatic leg pain: a case study," Acupuncture in Medicine, vol. 32, no. 5, pp. 418-422, 2014.

[25] E. Skorupska, M. Rychlik, W. Pawelec, A. Bednarek, and W. Samborski, "Intensive short-term vasodilation effect in the pain area of sciatica patients-case study," BMC Research Notes, vol. 7, no. 1, article 620, 2014.

[26] S. Doberenz, W. T. Roth, E. Wollburg, N. I. Maslowski, and S. Kim, "Methodological considerations in ambulatory skin conductance monitoring," International Journal of Psychophysiology, vol. 80, no. 2, pp. 87-95, 2011.

[27] Y. Nakatani, A Guide for Application of Ryodoraku Autonomous Nerve Regulatory Therapy, Chan's Books \& Products, Alhambra, Calif, USA, 1972.

[28] S. Kramer, D. Zaps, B. Wiegele, and D. Irnich, "Changes in electrical skin resistance at gallbladder 34 (GB34)," Journal of Acupuncture and Meridian Studies, vol. 1, no. 2, pp. 91-96, 2008.

[29] S. Grimnes and Ø. G. Martinsen, Bioimpedance and Bioelectricity Basics, Elsevier, New York, NY, USA, 2nd edition, 2008.

[30] A. P. Colbert, K. Spaulding, A. Larsen, A. C. Ahn, and J. A. Cutro, "Electrodermal activity at acupoints: literature review and recommendations for reporting clinical trials," Journal of Acupuncture and Meridian Studies, vol. 4, no. 1, pp. 5-13, 2011.

[31] H. N. Mayrovitz, M. Bernal, and S. Carson, "Gender differences in facial skin dielectric constant measured at $300 \mathrm{MHz}$," Skin Research and Technology, vol. 18, no. 4, pp. 504-510, 2012.

[32] H. N. Mayrovitz, S. Carson, and M. Luis, "Male-female differences in forearm skin tissue dielectric constant," Clinical Physiology and Functional Imaging, vol. 30, no. 5, pp. 328-332, 2010.

[33] T. Morimoto, "Variations of sweating activity due to sex, age and race," in The Physiology and Pathophysiology of the Skin (The Sweat Glands, Skin Permeation, Lymphatics, and the Nails, A. Jarrett, Ed., vol. 5, pp. 1655-1666, Academic, New York, NY, USA, 1978. 


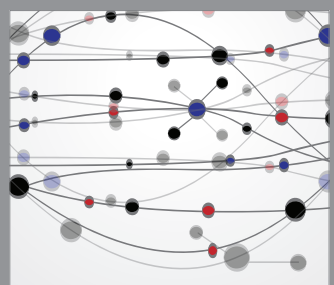

The Scientific World Journal
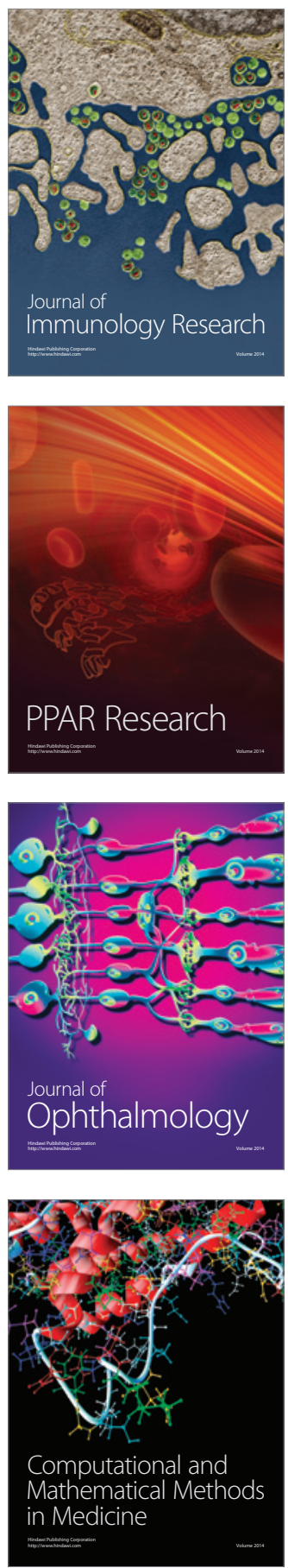

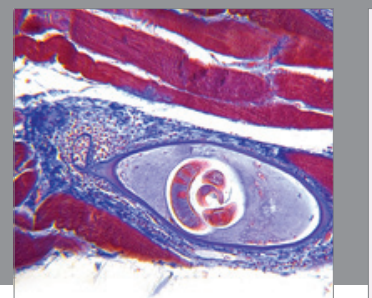

Gastroenterology

Research and Practice
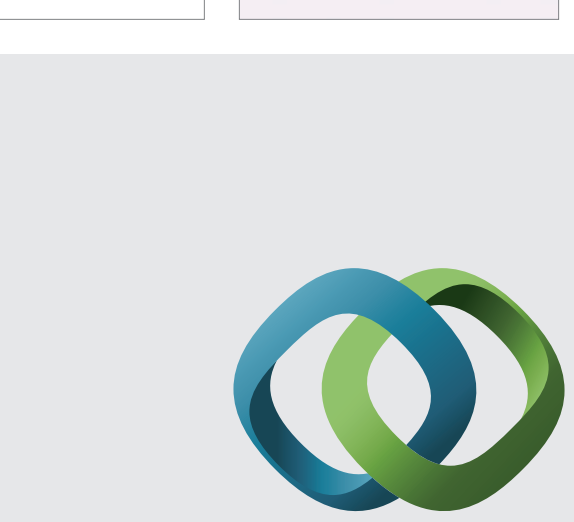

\section{Hindawi}

Submit your manuscripts at

http://www.hindawi.com
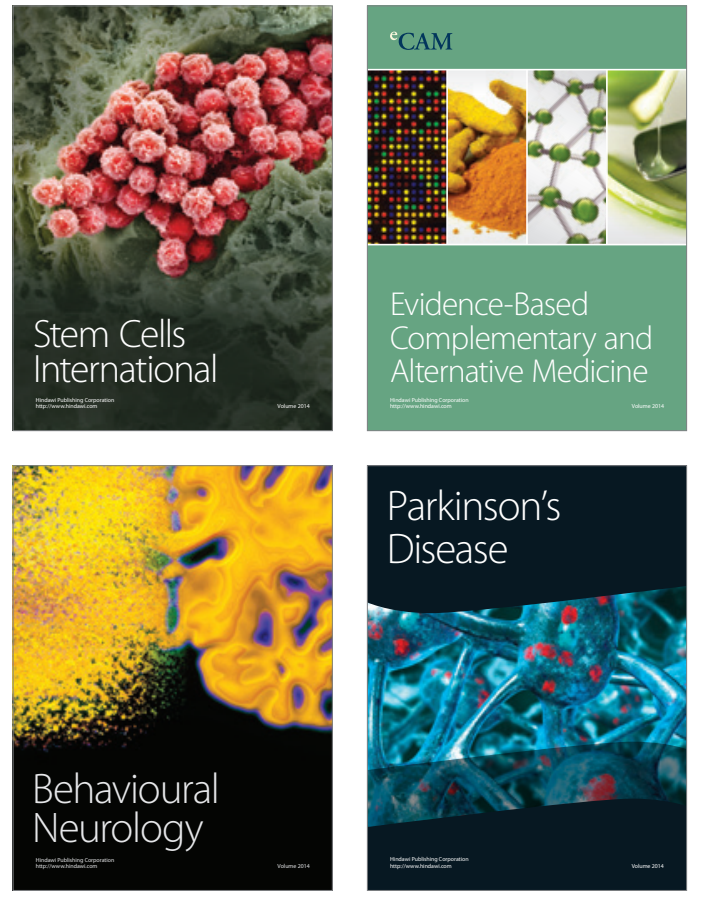
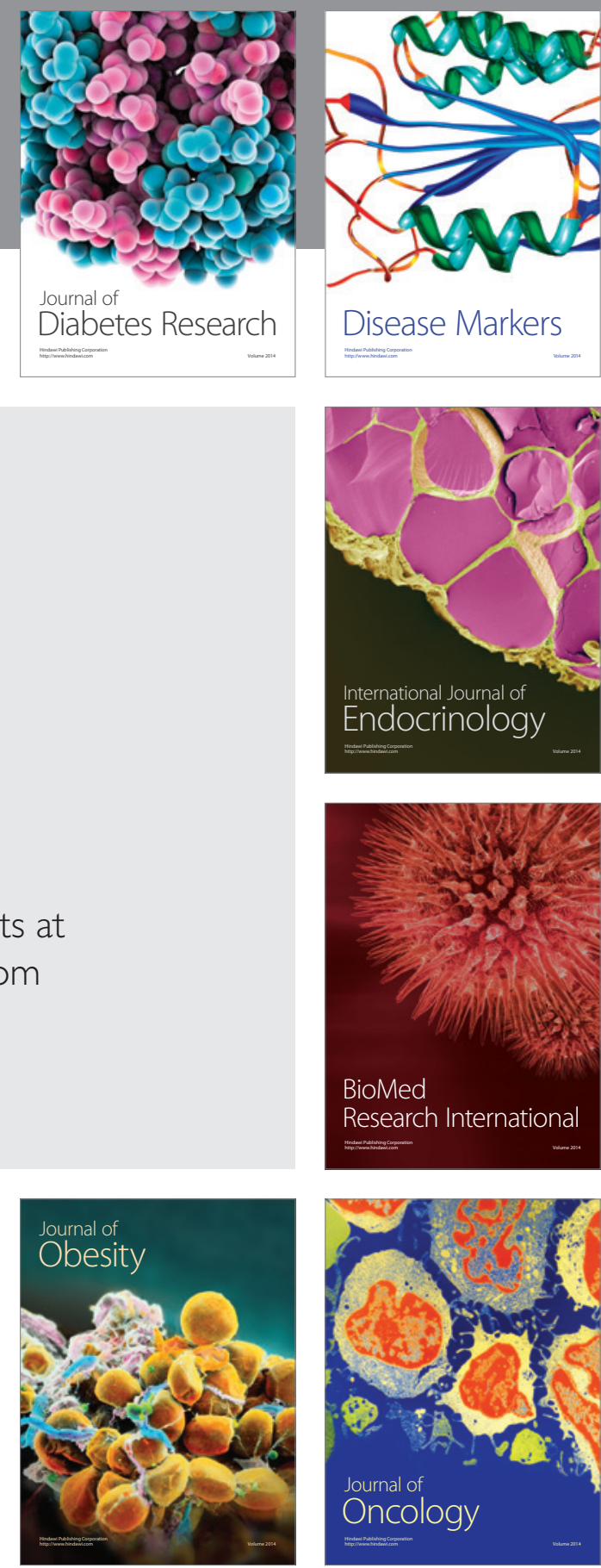

Disease Markers
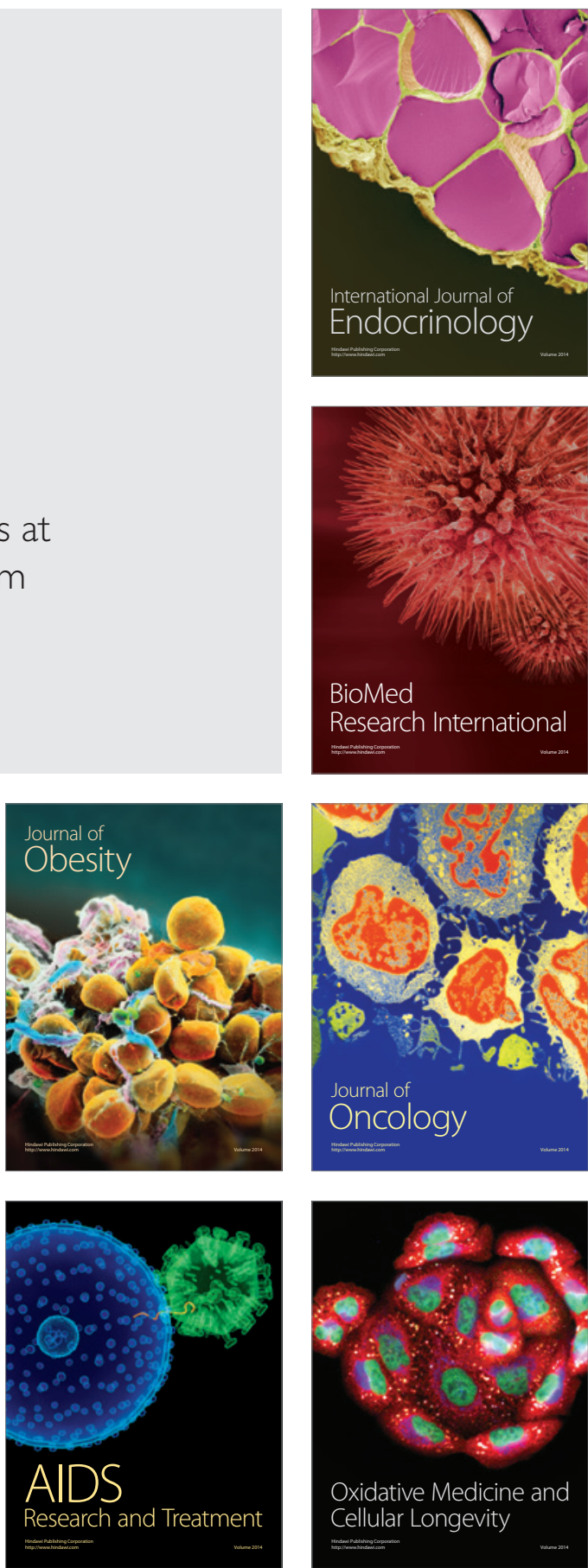